\title{
New Art For Crosshole Electromagnetic Tomography
}

\author{
CAO JUNXING $^{(1)}$ NIEZAIPING ${ }^{(2)}$ ZHU JESHOU $^{(1)}$ \\ (Chengdu University of Te'hnology. Chengdu 610059. China $)^{(1)}$ \\ (University of Electronic Science and Tedmology of China. Chengdu 610054. China ${ }^{\text {t2) }}$
}

\begin{abstract}
Crosshole Electromagnetic Tomography (CEMT) is a powerful geophysical exploration technique. Two new ray EM tomography strategies are proposed in this paper, one is named Average Multi-Frequency Absorption Tomography (AMFAT) and another Dual-Frequency Conductivity Tomography (DFCT). In AMFAT, the absorption tomography is done using the average of the data of different frequencies. The averaged data should be that measured at same configuration. In DFCT, the conductivity tomography is done using the following equation

$$
E_{1}-E_{2}=20 \log \left(\sqrt{f_{1} / f_{2}}\right)+8.6859 \sqrt{\pi \mu}\left(\sqrt{f_{2}}-\sqrt{f_{1}}\right) \int \hat{\sigma}(r) d r
$$

where $E_{1}, E_{2}$ are electric field intensities ( unit: $\mathrm{dB}$ ) measured at same geometry configuration in far field, $f_{1}, f_{2}$ are frequencies, $\hat{\sigma}=\sqrt{\sigma}, \sigma$ is the conductivity of the media detected.

DFCT is suitable for the medium with high conductivity and AMFAT is suitable for the medium with low conductivity. AMFAT can increase the S/N ratio. DFCT can markedly eliminate the image distortion rising from the approximation of the directional factor of dipole antenna used by that of a half wave-length dipole antenna.
\end{abstract}

Key Words: Tomography, dual-frequency EM tomography, multi-frequency EM absorption tomography, electromagnetic exploration

\section{INTRODUCTION}

Crosshole Electromagnetic Tomography (CET) is a powerful exploration technique. It has found many successful applications in engineering and environmental geology detection and mine resources exploration. For high frequency CET, ray absorption tomography is commonly used in the data interpretation. The absorption tomography equation can be written as (Cao et al., 1995)

$$
E=20 \lg \left(E_{0}\right)-8.687 \int_{R} \beta(r) d r+20 \lg \left(\frac{f(\theta) \sin \theta}{r_{0}}\right)
$$

where $E$ is the electric field intensity (unit: $\mathrm{dB}$ ) measured in the far field, $\beta(r)$ is the EM attenuation factor function of the earth medium, $\theta$ is the latitude angle, i.e. the angle between the dipole direction and 
the ray path, $r_{i 1}$ is the distance from the center point of the transmitter to the receiving antenna, $E_{0}$ and $f(\theta)$ are the primordial radiation intensity and directional factor of the transmitter. Assuming the medium is isotropic and homogeneous and the current in the center-fed dipole antenna is $I=I_{11} \sin [\alpha(I / 2-1:-1)] e^{-(k) \prime}$, then there will be (Wu and Xing. 1982)

$$
\begin{aligned}
& E_{0}=\frac{\omega \mu I_{0}}{4 \pi \alpha} \\
& f(\theta)=\frac{\cos [(\alpha l / 2) \cos \theta]-\cos (\alpha l / 2)}{\sin \theta}
\end{aligned}
$$

where $I_{0}$ is the feeding current at the center of the antenna, $l$ is the length of the antenna, $\omega$ is the angular frequency, $\alpha$ is the phase constant of the medium. It is well known that the phase constant $\alpha$ is a complex function of $\mu, \varepsilon, \sigma$ of the medium and $\omega$. So, the $\alpha$ in equation (2) and (3) is unknown and variable and so the $E_{0}$ and $f(\theta)$ in equation (1) cannot be precisely calculated out in common cases. It is a modus operandi that to treat the $E_{0}$ as constant and to approximate the $f(\theta)$ of the real dipole antenna by that of a perfect half wavelength dipole antenna. However, the approximation may result in some distortions of the image, such as the "pseudo cross abnormity". To eliminate the distortions, we have developed two new data interpretation strategies, one is named as "Dual Frequency Conductivity Tomography (DFCT)" and another the Average Multi-Frequency Absorption Tomography (AMFAT). DFCT is suitable for the medium with high conductivity and can markedly eliminate the image distortion rising from the approximation of the directional factor of the dipole antenna used by that of a half wavelength dipole antenna. AMFAT is suitable for the medium with low conductivity and can increase the $\mathrm{S} / \mathrm{N}$ ratio.

\section{DUAL FREQUENCY CONDUCTIVITY TOMOGRAPHY (DFCT)}

Theory

It is well known that

$$
\begin{aligned}
& \alpha=\omega \sqrt{\mu \varepsilon / 2} \sqrt{\sqrt{1+(\sigma / \omega \varepsilon)^{2}}+1} \\
& \beta=\omega \sqrt{\mu \varepsilon / 2} \sqrt{\sqrt{1+(\sigma / \omega \varepsilon)^{2}}-1}
\end{aligned}
$$

If the medium is good conductor, i.e. if $\sigma / \omega \varepsilon>1$, then, there will be

$$
\alpha=\beta \approx \sqrt{\omega \mu / 2} \sqrt{\sigma}=\sqrt{\omega \mu / 2} \hat{\sigma}(\hat{\sigma}=\sqrt{\sigma})
$$


where $\alpha$ and $\beta$ are the phase factor and attenuation factor of the earth medium respectively, $\sigma$ is the conductivity of the medium and $\hat{\sigma}$ is the square root conductivity of the earth medium.

Assuming the medium is isotropic and homogeneous and the current in the center-fed dipole antenna is $I=I_{1} \sin [\alpha(I / 2-|-|)] e^{-\mid(i) !}$, then the electric field intensity in far field excited by the transmitter can be written as (Wu and Xing, 1982)

$$
E=\frac{\omega \mu I_{0}}{4 \pi \alpha} \frac{e^{-\beta r}}{r_{0}} \frac{\cos [(\alpha l / 2) \cos \theta]-\cos (\alpha l / 2)}{\sin \theta}=\frac{\omega \mu I_{0}}{4 \pi \alpha} \frac{e^{-\beta r}}{r_{0}} f(\theta)
$$

where $l$ is the length of the transmitter.

Using two frequencies $f_{1}$ and $f_{2}$ to excite electromagnetic field at same configuration, two different electric intensities $E_{1}, E_{2}$ then will be measured. Expressing the $E_{1}, E_{2}$ as

$$
\begin{aligned}
& E_{1}=\frac{2 \pi f_{1} \mu I_{0}}{4 \pi \alpha_{1}} \frac{e^{-\beta_{1} r}}{r_{0}} f_{1}(\theta) \\
& E_{2}=\frac{2 \pi f_{2} \mu I_{0}}{4 \pi \alpha_{2}} \frac{e^{-\beta_{12} r}}{r_{0}} f_{2}(\theta)
\end{aligned}
$$

then we will have

$$
\frac{E_{1}}{E_{2}}=\frac{f_{1}}{\alpha_{1}} \cdot \frac{\alpha_{2}}{f_{2}} \cdot \frac{e^{-\beta_{1} r}}{e^{-\beta_{2} r}} \cdot \frac{f_{1}(\theta)}{f_{2}(\theta)}
$$

For small $\Delta f=f_{1}-f_{2}$, there will be $f_{1}(\theta) \approx f_{2}(\theta)$ and then the equation (10) can be written as

$$
\frac{E_{1}}{E_{2}}=\frac{f_{1}}{\alpha_{1}} \cdot \frac{\alpha_{2}}{f_{1}} \cdot e^{\beta_{2} r-\beta_{1} r}
$$

Substituting eq. (6) into eq. (11), it will be deduced that

$$
\frac{E_{1}}{E_{2}}=\sqrt{f_{1} / f_{2}} \cdot e^{\sqrt{\mu \pi}\left(\sqrt{f_{2}}-\sqrt{f_{1}}\right) \tilde{\sigma}}
$$

Treating the earth medium as continuous conductor and changing the unit of $E$ in equation (11) from $\mathrm{V} / \mathrm{m}$ to $\mathrm{dB}$, then we will have

$$
E_{1}-E_{2}=20 \log \left(\sqrt{f_{1} / f_{2}}\right)+8.6859 \sqrt{\pi \mu}\left(\sqrt{f_{2}}-\sqrt{f_{1}}\right) \int \hat{\sigma}(r) d r
$$

and

$$
\sum_{i}^{n} \int_{r_{\text {int }}}^{r_{\text {int }}} \hat{\sigma}(r) d r=c \cdot\left(E_{\jmath}-E_{2}\right)-C
$$




$$
c=\frac{1}{0.017258\left(\sqrt{f_{2}}-\sqrt{f_{1}}\right)} ; \quad C=20 \log \left(\sqrt{f_{1} / f_{2}}\right) \cdot c
$$

where $n$ is the total number of the discrete cells, $\hat{\sigma}(r)$ is the square root conductivity of the medium in the cell $i$, which is the function of the coordinate $r_{i n} . r_{\text {int }}$ are respectively the $i n-$ point and out-point coordinate of the ray at the cell $i$.

Equation (14) is the equation of Dual Frequency Conductivity Tomography. It is obvious that the conductivity distribution between the wells can be reconstructed without any need to calculate the directional factor $f(\theta)$ and the primordial radiation intensity $E_{0}$ of the transmitter antenna.

\section{Application conditions for DFCT}

DFCT has three connotative hypotheses:

(1) the conductivity $\sigma$ of the medium is independent on the work frequency;

(2) the attenuation factor $\beta$ of the medium is dependent on the work frequency, i.e. $\beta$ will enlarge along with the increasing of the work frequency;

(3) the increasing of the work frequency only result in a small change in the directional factor $f(\theta)$

For a relatively low frequency $(<100 \mathrm{MHz})$, hypothesis (1) is almost true in earth medium. However, equation (4), (5) and (3) show that the hypothesis (2) and (3) are some conflicting in common cases, i.e. if $\beta$ is dependent on the frequency, $f(\theta)$ will also be dependent on the frequency. So attention should be paid to both hypothesis (2) and (3) when work frequencies are chosen.

Figure 1 shows some curves that indicate the relationship between the attenuation factor (or called absorption coefficient) $\beta$ and the work frequency $f$ and the conductivity $\sigma$ of the medium. The corresponding phase factor curves are also shown in the figure 1.The $\mu_{r}$ and $\varepsilon_{r}$ of the medium are assumed to be same in all three cases. 


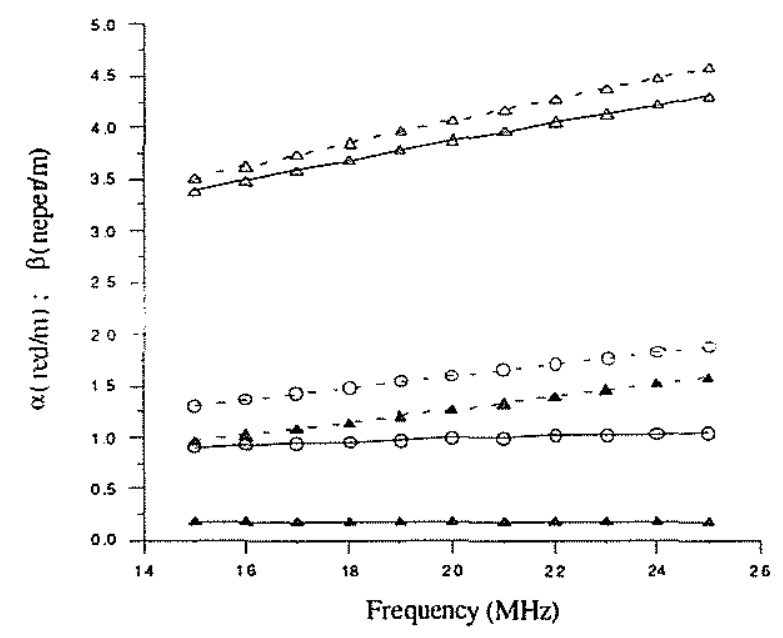

Figure 1. Curves showing the relationship between the attenuation factor ( $\beta$ : real line), phase factor ( $\alpha$ : dashed) and work frequency and the conductivity of the medium.

$\therefore, 0,{ }^{*}$ indicate different conductivity, they are $0.02,0.002,0.0003 \mathrm{~S} / \mathrm{m}$ in urn. $\mu_{r}$ and $\varepsilon_{r}$ of the medium are 1 and 9 respectively in all three cases.

Figure 1 shows that the relationship between the attenuation factor and the work frequency is dependent on the conductivity of the medium. If the medium is high conductivity, its attenuation factor will be markedly dependent on the work frequency. In other words, DFCT should be used in the case that the detected medium is high conductivity $(>0.01 \mathrm{~S} / \mathrm{m})$.

The curves in figure 2 show the relationship between the directional factor $f(\theta)$ of the transmitter and its work frequency $f$ and the conductivity $\sigma$ of the medium. (a), (b) and (c) in figure 2 correspond to different electromagnetic parameters, which are as follows: (a) $\sigma=0.02 \mathrm{~S} / \mathrm{m}, \varepsilon_{r}=9, \mu_{r}=1$; (b) $\sigma=$ $0.002 \mathrm{~S} / \mathrm{m}, \varepsilon_{r}=9, \mu_{r}=1 ;$ (c) $\sigma=0.0003 \mathrm{~S} / \mathrm{m}, \varepsilon_{r}=9, \mu_{r}=1$. The curves without any mark in each group correspond to different frequencies, from the bottom to the top, they correspond to $15,16,17,18,19,20,21$, $22,23,24,25 \mathrm{MHz}$ in tum. The curve with " $\mathrm{A}$ " mark is the $f(\theta)$ curve of a half wavelength dipole antenna, which is commonly used in the electromagnetic ray absorption tomography (Cao et. al., 1995). Figure 2 shows that the relationship between the directional factor and the work frequency is also dependent on the conductivity of the medium. It is also showed that, for the medium with higher conductivity, the real $f(\theta)$ curve of the transmitter may be far away from that of a half wavelength antenna with same length, as shown in group (a). It is obvious that, for high conductivity medium cases, DFCT can weaken the error in the calculation of the $f(\theta)$ and so can eliminate the image distortion rising from the error.

\section{AVERAGE MULTI-FREQUENCY ABSORPTION TOMOGRAPHY (AMFAT)}

It is common that for the data measured in CET to be contaminated by various noises. If we can find out a 
reasonable warrant to average the data of different frequencies, then we find one simple means to weaken the noises that are dependent on frequency. The warrant is in Figure 1. Figure 1 shows that the attenuation factor and phase factor of low conductivity medium are almost independent on the frequency.
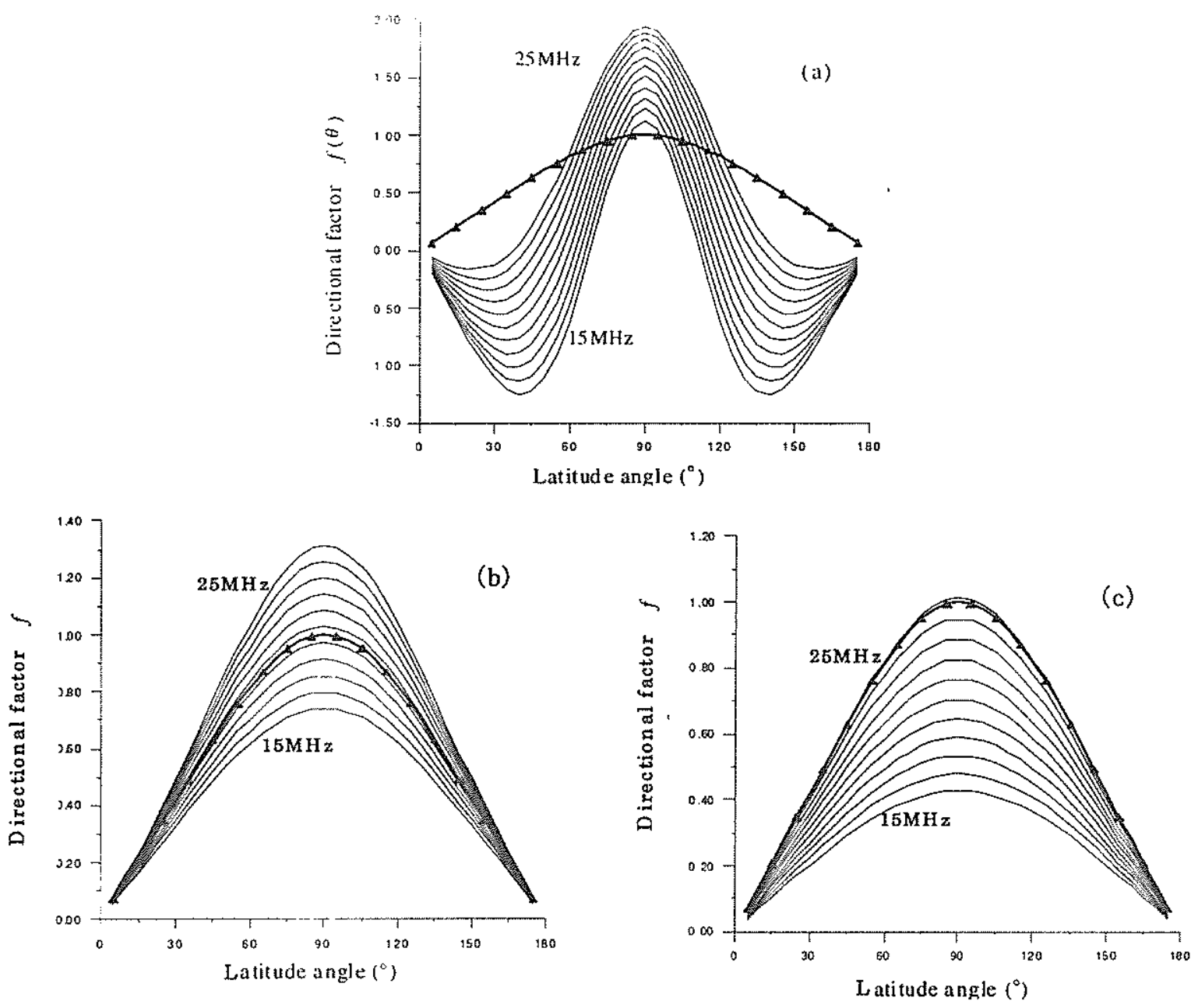

Figure 2 Curves showing the relationship between the directional factor of the dipole antenna and its work frequency and the conductivity of the medium

The curves without mark in each group indicate different frequencies. The curves with " $\Delta$ " mark indicate the directional factor of a half wavelength dipole antenna. The electromagnetic parameter of (a), (b) and (c) are as follows:

(a) $\sigma=0.02 \mathrm{~S} / \mathrm{m} \quad \varepsilon_{r}=9 \quad \mu_{r}=1$; (b) $\sigma=0.002 \mathrm{~S} / \mathrm{m} \quad \varepsilon_{r}=9 \quad \mu_{r}=1$; (c) $\sigma=0.0003 \mathrm{~S} / \mathrm{m}, \varepsilon_{r}=9 \mu_{r}=1$

It means that we can use the average of the data of different frequencies as single frequency data to do absorption tomography. Let

$$
E_{a v}=\sum_{i=1}^{i=n} E_{i}
$$


where $E_{1}, E_{2}, \cdots, E_{n}$ are $n$ measured data sets, which are correspond to $n$ different frequencies $f_{1}, f_{2}, \cdots, f_{n}$. Then the equation of AMFAT (Average Multi-Frequency Absorption Tomography) will be

$$
E_{i n}=-8.687 \int_{k} \beta(r) d r+20 \lg \left(E_{11}\right)+20 \lg \left(\frac{f(\theta) \sin \theta}{R}\right)
$$

where the variables have the same means as in equation (1). It is obvious that equation (15) is same in form with ordinary absorption tomography equation (1). However, it should be used only for low conductivity medium case.

\section{FIELD EXAMPLES}

DFCT and AMFAT have been successfully applied in real data interpretations. Some examples are as follows.

Figure 3 shows a conductivity tomogram (a) and an absorption tomogram (b). The first is obtained by DFCT using the $18 \mathrm{MHz}$ and $22 \mathrm{MHz}$ data sets; the latter is obtained by the ordinary ray absorption tomography using the $20 \mathrm{MHz}$ data set. Both tomograms are for same profile. The goal of the investigation is to determine the extent of fine sand layers discovered by the drilling and detect whether there are other fine sand layers in the investigation area

It is obvious that there are some differences between the two tomograms. According to the drilling records, the conductivity tomogram is more consistent with the real geology profile than the absorption tomogram is. The abnormally low conductivity belt on the top of the conductivity tomogram is the response of the dry loose clay and grit layer. The abnormal high conductivity belts between the depths of $24 \mathrm{~m}$ and $28 \mathrm{~m}$ are the responses of three lentoid fine sand layers, one of which is identified by the EM tomography. In sum, DFCT proposed in this paper could give fine tomogram of a profile investigated.

Figure 4 shows two absorption tomograms reconstructed by AMFAT using average of data of 7 different frequencies (from $15 \mathrm{MHz}$ to $21 \mathrm{MHz}$ with an interval of $1 \mathrm{MHz}$ ). They are obtained in an investigation of karst. The high absorption zones $(>0.2 \mathrm{Neper} / \mathrm{m})$ in the tomograms are interpreted as karst.. In fact, AMFAT was proposed out in the interpretation of the data measured in the investigation. For poor field condition and some troubles in the instruments, we did not obtained good data in the field works. In the interpretation of the data, ordinary absorption tomography often fails to give reasonable results. To obtain reasonable tomograms from the poor data, we develop AMFAT and find successful application. 


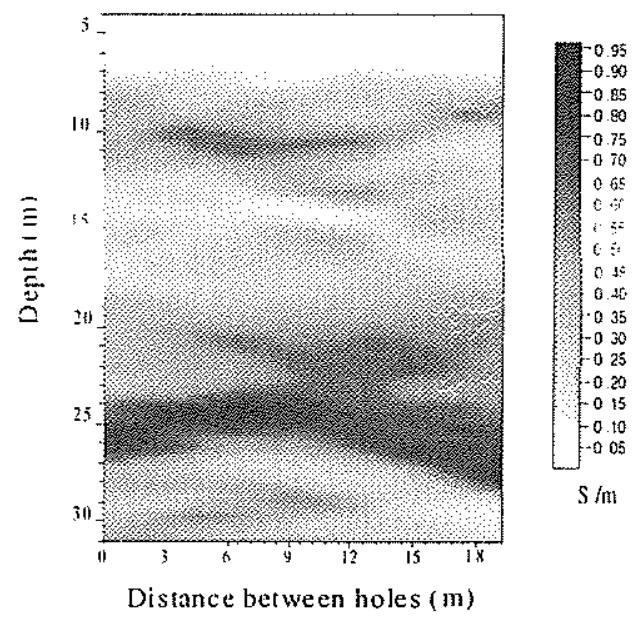

(a)

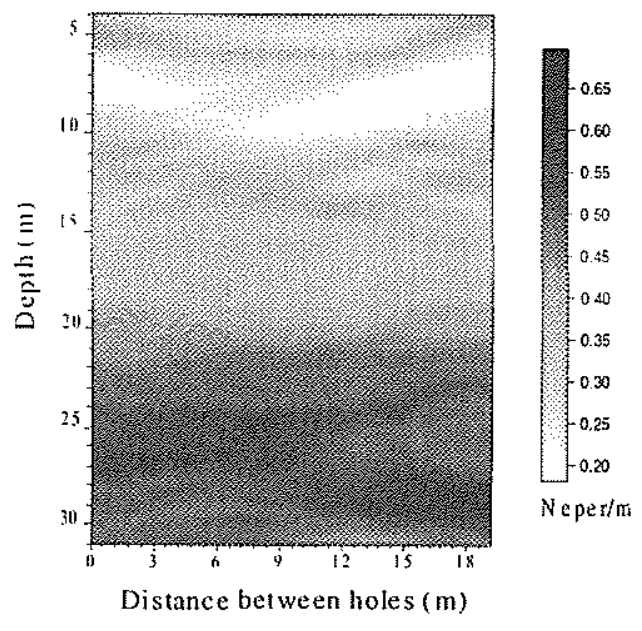

(b)

Figure 3 Conductivity tomogram (a) and absorption tomogram(b) of a profile
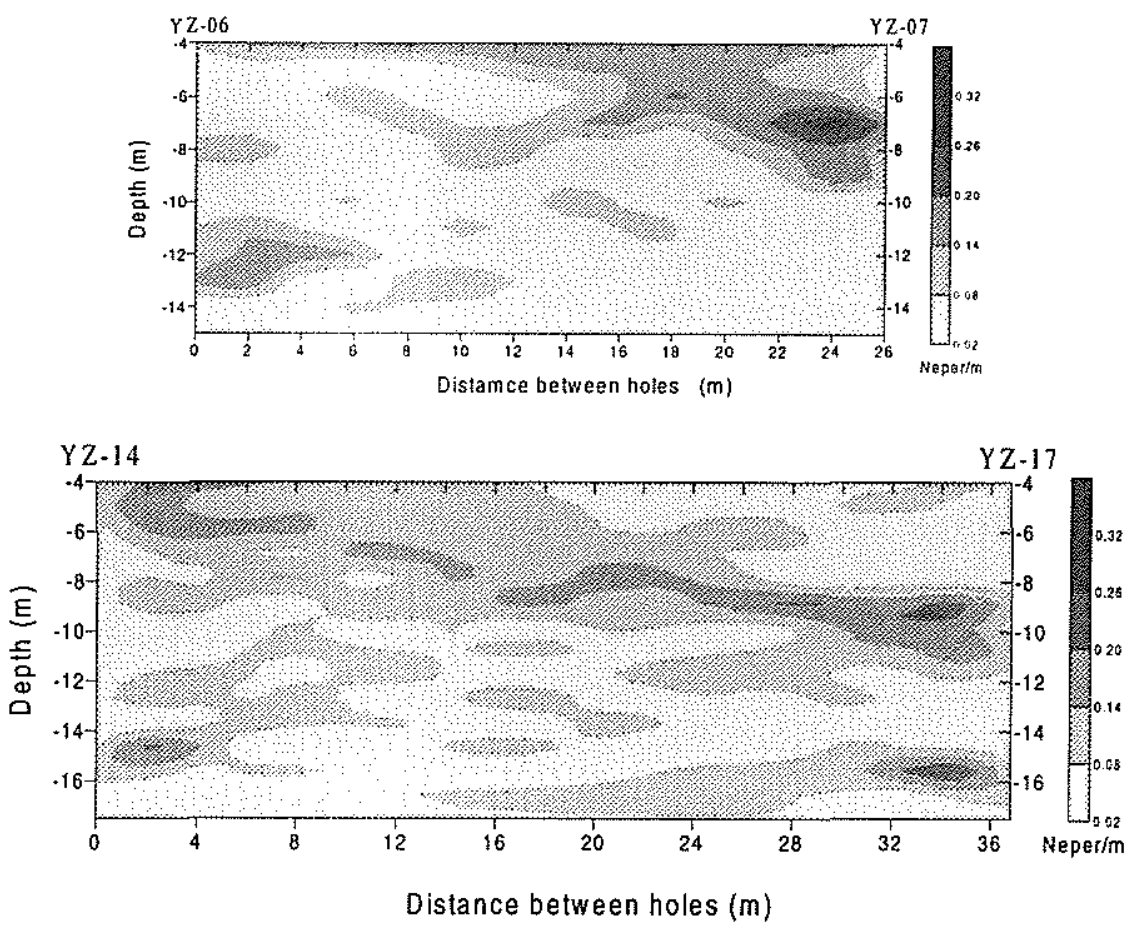

Figure 4 EM absorption tomograms reconstructed by AMFAT 


\section{CONCLUSION}

Crosshole Electromagnetic Tomography (CET) is a powerful tool to image fine geostructures. Both Dual Frequency Conductivity Tomography (DFCT) and Average Multi-Frequency Absorption Tomography (AMFAT) proposed in this paper can give more precise image of a profile detected than ordinary absorption tomography does. DFCT can be treat as a new ray tomography strategy and AMFAT is only an improved version of ordinary absorption tomography. DFCT should be used for high conductivity medium cases and AMFAT for low conductivity medium cases.

\section{ACKNOWLEDGEMENTS}

We would like to thank Prof. Yang Zhongqiong, Dr. Cao Xiaolin, Eng. Li Jian and Prof. Cao Jiaming Fu Weiyi and other follows for their kind help in field works. The work was partly supported by the National Nature Science Foundation of China (NSFC) under grant No. 49774222 and the State Key Lab. of Oil and Gas Reservoir Geology and Exploitation under grant No. PLC9610.

\section{REFERENCES}

Cao Junxing, Zhu Jieshou and Yan Zhongqiong. 1995, Fracture Imaging Using Electromagnetic Crosshole Tomography. In: Geotomography -Fracture Imaging, SEGJ, pp220-287.

Wu Yiren, Xing Fengtong (editors), 1982, Borehole Electromagnetic method, Geological Press, Beijing, (in Chinese)

Rui Feng, Hainam Zhou, Yulu Tao and Jingtao Zhang, 1995, Application of electromagnetic tomography in fractured zones prospecting: Geotomography -Fracture Imaging, pp228-235. 